\title{
Construction of tilted algebras
}

Dieter Happel and Claus Michael Ringel

Let $A$ be a finite dimensional algebra. The (finite dimensional) A-module $T_{A}$ is said to be a tilting module provided it satisfies the following three properties:

(1) There is an exact sequence $\circ \longrightarrow \mathrm{P}_{\mathrm{A}}^{\prime \prime} \longrightarrow \mathrm{P}_{\mathrm{A}}^{\prime} \longrightarrow \mathrm{T}_{\mathrm{A}} \longrightarrow$ o with $\mathrm{P}^{\prime}, \mathrm{P}^{\prime \prime}$ projective (thus proj. dim. $\left(\mathrm{T}_{\mathrm{A}}\right) \leq 1$ ).

(2) $\operatorname{Ext}^{1}\left(\mathrm{~T}_{\mathrm{A}}, \mathrm{T}_{\mathrm{A}}\right)=0$.

(3) There is an exact sequence $o \longrightarrow \mathrm{A}_{\mathrm{A}} \longrightarrow \mathrm{T}_{\mathrm{A}}^{\prime} \longrightarrow \mathrm{T}_{\mathrm{A}}^{\prime \prime} \longrightarrow \mathrm{o}$ with $\mathrm{T}^{\prime}, \mathrm{T}^{\prime \prime}$ being direct sums of direct summands of $T_{A}$ (thus, $T_{A}$-codim. $\left(A_{A}\right) \leq 1$ ).

The finite dimensional algebras of the form End $\left(T_{A}\right)$, where ${ }_{A}$ is a tilting module, with $A$ a finite dimensional hereditary algebra, are called the tilted algebras. The main interest in tilted algebras comes from the fact that any finite dimensional algebra with a faithful indecomposable module and with Auslander-Reiten quiver without oriented cycle is a tilted algebra. We will recall some properties of tilted algebras in Section 1, but refer for the proofs to [5].

We will show that any hereditary algebra A has only finitely many tilting modules with basic endomorphism ring of finite representation type (Proposition 2.1). In fact, the proof will provide an inductive procedure for obtaining all these algebras explicitly. The same method also shows that in case $A$ is in addition tame, there are only finitely many basic algebras which are endomorphism rings of tilting modules. Besides those of finite type, one also obtaines concealments and domestic regular or coregular enlargements of concealed hereditary algebras (see [6]), the finite type being characterized by the fact that the tilting module has both nonzero preprojective and non-zero preinjective direct summands. The essential properties of tilting modules over tame hereditary algebras without preinjective or preprojective direct summands are collected in Proposition 3.2 and 3.2*. Finally, we want to exhibit some special hereditary algebras $A$ in detail and list corresponding tilting modules, namely we consider hereditary algebras of type $A_{n}, E_{6}$, $\tilde{A}_{n}$ and $\tilde{E}_{6}$. 


\section{Report on tilted algebras}

Let $A$ be a hereditary algebra. In this case, it is rather easy to check whether a given module is a tilting module. Namely, $\mathrm{T}_{\mathrm{A}}$ is a tilting module if and only if $\operatorname{Ext}^{1}\left(\mathrm{~T}_{\mathrm{A}}, \mathrm{T}_{A}\right)=0$, and the number of (isomorphism classes of) indecomposable direct summands of $T_{A}$ is equal to the number of simple A-modules.

Now let $T_{A}$ be a tilting module. We consider the following two full subcategories: $T\left(\mathrm{~T}_{\mathrm{A}}\right)$ will denote the full subcategory of all modules generated by $\mathrm{T}_{\mathrm{A}}$. Note that a module $X_{A}$ is generated by $T_{A}$ if and only if $\operatorname{Ext}_{A}^{1}\left(T_{A}, X_{A}\right)=0$. And, $F\left(T_{A}\right)$ will denote the full subcategory of all modules cogenerated by $\tau_{A}$, where $\tau$ is the Auslander-Reiten translation $\tau=\mathrm{D} T r$. A module $\mathrm{X}_{\mathrm{A}}$ is cogenerated by $\mathrm{T}_{\mathrm{A}}$ if and only if $\operatorname{Hom}_{A}\left(T_{A}, X_{A}\right)=0$. Always, the pair $\left(T\left(T_{A}\right), F\left(T_{A}\right)\right)$ forms a torsion theory.

Consider now the tilted algebra $B=\operatorname{End}\left(\mathrm{T}_{A}\right)$. In $M_{B}$, there is a splitting torsion theory $(X, Y)$ such that $X$ is equivalent, as a category, to $F\left(T_{A}\right)$, and $Y$ is equivalent to $T\left(T_{A}\right)$. In fact, the functor $\operatorname{Hom}_{A}\left({ }_{B} T_{A},-\right)$ has as image just $y$, and its restrictions to $T\left(T_{A}\right)$ furnishes an equivalence $T\left(T_{A}\right) \longrightarrow y$, as Brenner and Butler have shown. Similarly, the functor $\operatorname{Ext}_{A}{ }_{A}\left({ }_{B} T_{A},-\right)$ has as image just $x$, and its restriction to $F\left(\mathrm{~T}_{\mathrm{A}}\right)$ furnishes an equivalence $F\left(\mathrm{~T}_{\mathrm{A}}\right) \rightarrow X$. Since the torsion theory $(X, Y)$ is splitting, we see that we obtain any indecomposable B-module either in the form $\operatorname{Hom}_{A}\left({ }_{B} T_{A}, M_{A}\right)$ with $M_{A} \in T\left(T_{A}\right)$, or in the form $\operatorname{Ext}_{A}^{1}\left({ }_{B} T_{A}, M_{A}\right)$ with $M_{A} \in F\left(T_{A}\right)$. The indecomposable modules of the form $\operatorname{Hom}_{A}\left({ }_{B} T_{A}, M_{A}\right)$ all have projective dimension 0 or 1 , those of the form $\operatorname{Ext}_{A}{ }_{A}\left({ }_{B} T_{A}, M_{A}\right)$ have projective dimension 1 or 2. In particular, the global dimension of a tilted algebra always is $\leq 2$.

As an example, consider the path algebra A of the quiver $0 \rightarrow 0 \rightarrow 0+0<0$ of type $E_{6}$. The Auslander-Reiten quiver of $A$ has the following form, the indecomposables being denoted by their dimension type:

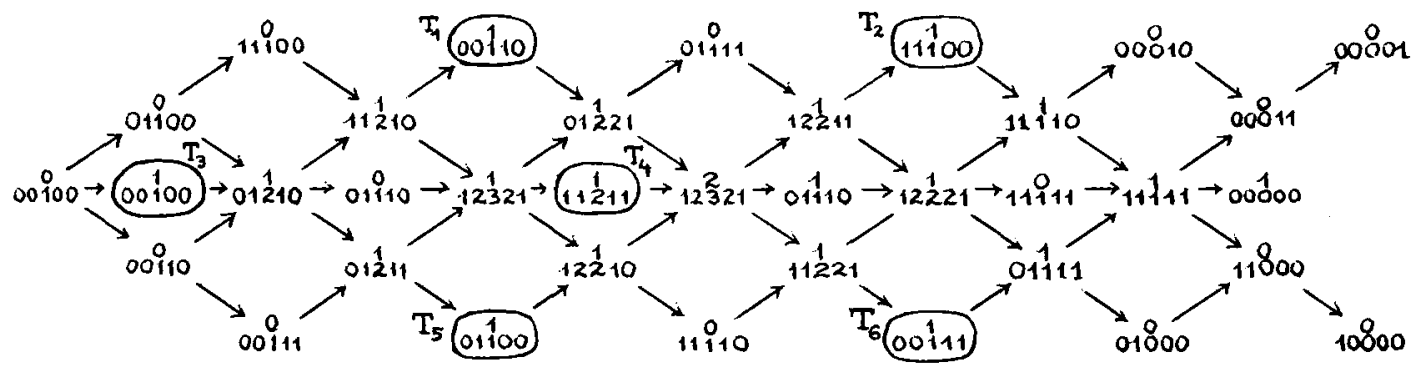

6

we have marked one tilting module $\mathrm{T}_{\mathrm{A}}=\underset{i=1}{\oplus} \mathrm{T}$. Let us indicate the subcategories $F\left(T_{A}\right)$ by $\because$, and $T\left(T_{A}\right)$ by $Q T D !=1$ 


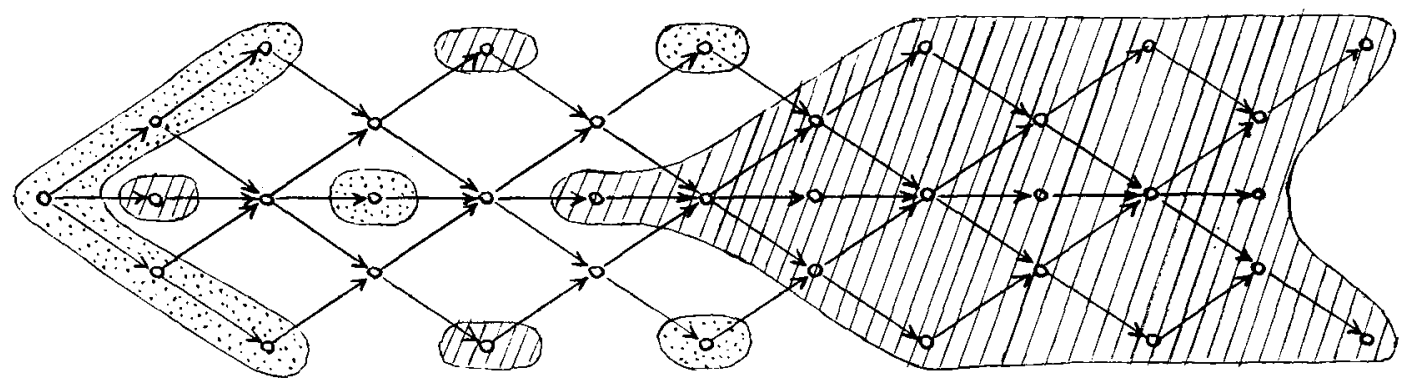

Now $B=$ End $\left(T_{A}\right)$ is the algebra given by the quiver

$\underset{\substack{q \rightarrow 0+q \\ 0 \rightarrow 0+0}}{\substack{+0}}$ with al1 commutativity relations, its Auslander-Reiten quiver is as follows (here $x$ is marked by

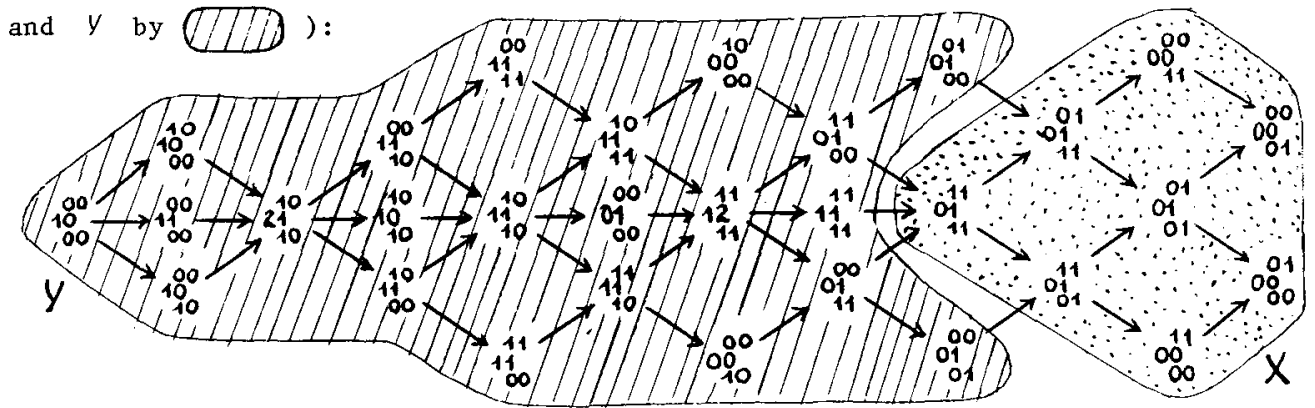

There is a rather useful criterion in order to decide that an algebra $B$ is a tilted algebra. Namely, assume that the Auslander-Reiten quiver $\Gamma(B)$ of $B$ has a component $C$ which contains no oriented cycles, such that any module in $C$ is of the form $\tau^{-n} P$ for some $n \in \mathbb{N}$ and some projective module $P$ (then $C$ may be called a "preprojective" component; in particular, if $B$ is connected and of finite representation type, then $\Gamma(B)$ itself is a preprojective component if and only if $\Gamma(B)$ has no oriented cycles.) Now, if $C$ contains an indecomposable faithful module $N_{B}$, then $B$ is a tilted algebra: there exists a hereditary algebra A, a tilting module $T_{A}$, and an indecomposable injective module $I_{A}$ such that $\mathrm{B}=\operatorname{End}\left(\mathrm{T}_{\mathrm{A}}\right)$, and $\mathrm{N}_{B}=\operatorname{Hom}_{A}\left(\mathrm{~B}_{\mathrm{A}}, \mathrm{I}_{\mathrm{A}}\right)$. In fact, usually there are many different choices possible for $A, T_{A}$ and $I_{A}$, as we will see below.

A very special kind of a tilting module is the slice module of a finite complete slice in a component $C$ of $\Gamma(B)$ which contains all indecomposable projective modules. Recall that a set $U$ of modules in $C$ is said to form a complete slice provided the following three conditions are satisfied

(i) For any $x$ in $C$, there exists a unique module of the form $\tau^{2} X$ with $z \in \mathbf{z}$ which belongs to $U$.

(ii) If $x_{0} \longrightarrow x_{1} \rightarrow \ldots \rightarrow x_{r}$ is a chain of non-zero maps and indecomposable modules, and both $x_{0}, x_{r}$ belong to $u$, then all $x_{i}$ belong to $u$.

(iii) There is no oriented cycle $\mathrm{U}_{\mathrm{o}} \rightarrow \mathrm{U}_{1} \rightarrow \ldots \rightarrow \mathrm{U}_{\mathrm{r}} \rightarrow \mathrm{U}_{\mathrm{O}}$ of irreducible maps with all $v_{i}$ in $u$. 
Note that the endomorphism ring of the slice module of a finite complete slice in a component which contains all indecomposable projective modules always is hereditary. In case we start with a complete slice in the preprojective component of a hereditary algebra $A$, say with siice module $U_{A}$, and $B=\operatorname{End}\left(U_{A}\right)$, then we will say that $B$ is obtained from $A$ by an admissible change of orientation.

Such a change of orientation can be established also by a sequence of the following operation: Let $\mathrm{P}(1), \ldots, \mathrm{P}(\mathrm{n})$ be the indecomposable projective A-modules, and assume $P(1)$ is simple. Let $U_{A}=\tau^{-1} P(1) \oplus \underset{i=2}{\oplus} P(i)$. Then $U_{A}$ is the slice module of a complete preprojective slice, and the quiver of End $\left(U_{A}\right)$ is obtained from that of $A$ by reversing all arrows involving the point $P(1)$ (Recall that the quiver of a hereditary algebra $A$ is obtained from the full subquiver of $\Gamma(A)$ consisting of the indecommosable projective modules by reversing all arrows). The corresponding functor $\operatorname{Hom}_{A}\left({ }_{B} U_{A},-\right): M_{A} \longrightarrow M_{B}$ usually is called a (Bernstein-Gelfand-Ponomarev) reflection functor (see [1]). Also note that in case the quiver of the hereditary algebra $A$ is a tree $\Delta$ then any change of orientation of $\Delta$ can be realized by a sequence of reflection functors, thus is admissible.

Lemma: Let $A$ be hereditary, $T_{A}$ a tilting module, and $B=$ End $\left(T_{A}\right)$. Let $I_{A}$ be indecomposable injective, and assume $N_{B}=\operatorname{Hom}_{A}\left({ }_{B} T_{A}, I_{A}\right)$ is a faithful B-module. Let $A^{\prime}$ be obtained from $A$ by an admissible change of orientation. Then there exists a tilting module $\mathrm{T}^{\prime} \mathrm{A}^{\prime}$, and an indecomposable injective $\mathrm{A}^{\prime}$-module $I_{A^{\prime}}^{\prime}$ such that $B=\operatorname{End}\left(T_{A^{\prime}}^{\prime}\right)$ and $N_{B}=\operatorname{Hom}_{A^{\prime}}\left(B_{A^{\prime}}^{\prime}, I_{A^{\prime}}^{\prime}\right)$.

Proof: Since $N_{B}$ is faithful, we have $\operatorname{Hom}_{B}\left(Q_{B}, N_{B}\right) \neq 0, \operatorname{Hom}_{B}\left(N_{B} J_{B}\right) \neq 0$ for any indecomposable projective module $Q_{B}$ and any indecomposable injective module $\mathrm{J}_{B}$. Consider the complete slice $U$ consisting of all indecomposable modules $X_{B}$ which have a path of irreducible maps from $X_{B}$ to $N_{B}$ such that any such path is sectional. It has been shown in [5] 7.2 that the corresponding siice module $U_{B}$ is a tilting module, and $A_{0}=\operatorname{End}\left(U_{B}\right)$ is hereditary with a unique simple injective module $I_{0}$ such that $\mathrm{N}_{B}=\operatorname{Hom}_{\mathrm{A}_{0}}\left({ }_{B} D(U) A_{0}, I_{0}\right)$. Starting from $U$, we construct inductively various slices, always replacing an indecomposable module $X_{B}$ which is a source in one slice (and $x_{B} \neq N_{B}$ ), by $\tau^{-1} x_{B}$. Note that always $\tau^{-1} X_{B} \neq 0$, due to the fact that $N_{B}$ is faithful. The corresponding slice modules have as endomorphism rings just hereditary algebras obtained from $A_{0}$ (and therefore also from A) by all possible admissible changes of orientations.

We note the following consequence: In case the underlying graph of the quiver of $A$ is a tree, then we can construct all possible tilted algebras End $\left(\mathrm{T}_{A}\right)$ which are of finite representation type and have an indecomposable faithful representation, by considering instead of $A$ any fixed algebra obtained from $A$ by a change of orientation. 
Finally, we remark that in case $B$ is of finite representation type and $\Gamma(B)$ has no oriented cycles, then an indecomposable module $N_{B}$ is faithful if and only if it satisfies the following rather weak condition: $\operatorname{Hom}_{B}\left(P_{B}, N_{B}\right)=0$ for a projective module $\mathrm{P}_{\mathrm{B}}$ imp1ies $\mathrm{P}_{\mathrm{B}}=0$. 


\section{Tilted algebras of finite representation type}

The following proposition shows that for a fixed hereditary algebra A, there are only finitely many multiplicity-free tilting modules $T_{A}$ with End $\left(T_{A}\right)$ of finite representation type. (Recall that a module $M_{A}$ is called multiplicity-free, if for a decomposition $M_{A}=\oplus M_{i}$ with $M_{i}$ indecomposable, these modules $M_{i}$ are pairwise non-isomorphic, and this happens if and only if $\operatorname{End}\left(M_{A}\right)$ is basic). Thus we can derive from A only a finite number of Morita equivalence classes of algebras of finite representation type as endomorphism rings of tilting modules. In fact, the proof of the proposition provides an inductive procedure in order to obtain all these algebras.

Proposition (2.1) Let $A$ be a hereditary algebra. There are only finitely many multiplicity-free tilting modules $T_{A}$ with End $\left(T_{A}\right)$ of finite representation type, and any such tilting module has both indecomposable preprojective and indecomposable preinjective direct summands.

The proof will use the following lemma which is of use also in other situations:

Lemma (2.2) Let $A$ be a connected hereditary algebra of infinite representation type, and $T_{A}$ a tilting module. Then: $T_{A}$ has no non-zero preprojective direct summand if and only if all preprojective modules belong to $F\left(T_{A}\right)$. Also, $\mathrm{T}_{\mathrm{A}}$ has no non-zero preinjective direct summand if and only if all preinjective modules belong to $T\left(T_{A}\right)$.

Proof of the lemma. A module $M_{A}$ belongs to $F\left(T_{A}\right)$ iff $\operatorname{Hom}_{A}\left(T_{A}, M_{A}\right)=0$. Now for $M_{A}$ preprojective, and $\operatorname{Hom}_{A}\left(X_{A}, M_{A}\right) \neq 0$, it follows that $X_{A}$ has a nonzero preprojective direct summand. Thus, if $\mathrm{T}_{\mathrm{A}}$ has no non-zero preprojective direct summand, then all preprojective modules belong to $F\left(\mathrm{~T}_{\mathrm{A}}\right)$. Conversely, if $T(i)$ is an indecomposable preprojective direct summand of $T_{A}$, then clearly $\operatorname{Hom}\left(\mathrm{T}_{\mathrm{A}}, \mathrm{T}(\mathrm{i})_{\mathrm{A}}\right) \neq \mathrm{o}$, thus $\mathrm{T}(\mathrm{i})$ is not in $F\left(\mathrm{~T}_{\mathrm{A}}\right)$. Similarly, a module $\mathrm{M}_{\mathrm{A}}$ belongs to $T\left(T_{A}\right)$, if and only if $0=\operatorname{Ext}^{1}\left(T_{A}, M_{A}\right)=D \operatorname{Hom}_{A}\left(M_{A}, T T_{A}\right)$. If $M_{A}$ is preinjective, and $\operatorname{Hom}\left(\mathrm{M}_{\mathrm{A}}, \mathrm{Y}_{\mathrm{A}}\right) \neq 0$, then $\mathrm{Y}_{\mathrm{A}}$ has a non-zero preinjective direct summand. Thus, if $T_{A}$ has no non-zero preinjective direct sumand, the same is true for ${ }^{T} T_{A}$, and therefore all preinjective modules are in $T\left(T_{A}\right)$. Conversely, assume $T(j)$ is an indecomposable preinjective direct summand of $T$. Since $A$ is connected and of infinite representation type, $T(j)$ is not projective, thus $\tau T(j)$ is non-zero. It is a preinjective module with $\operatorname{Hom}\left(\tau \mathrm{T}(j)_{\mathrm{A}}, \mathrm{TT}_{\mathrm{A}}\right) \neq 0$, thus does not belong to $T\left(\mathrm{~T}_{\mathrm{A}}\right)$.

Proof of the proposition: The second assertion is an immediate consequence of the previous lemma. Let us now prove the first assertion by induction on the number $n$ of simple A-modules. For $n=1$, there is just one tilting module. Now let $A$ be an algebra with $n>1$ simple modules, let $P(1), \ldots, P(n)$ be the 
indecomposable projective A-module.

First, let us construct all tilting modules with a non-zero projective direct summand. For any non-empty subset $I \subseteq\{1, \ldots, n\}$, let $P(I)=\underset{i \in I}{\oplus}(i)$. Note that there exists an idempotent $e(I)$ of $A$ such that $P(I) \approx A e(I)$. Let $A\left(I^{\prime}\right)=A /<e>$, with $<e>$ the twosided ideal generated by e. Consider $M_{A}\left(I^{\prime}\right)$ as the full subcategory of $M_{A}$ of all modules $X_{A}$ satisfying $\operatorname{Hom}_{A}\left(P(I){ }_{A}, X_{A}\right)=0$. Now $A\left(I^{\prime}\right)$ is hereditary, and has a smaller number of simple modules, thus, by induction, there are only finitely many multiplicity-free tilting modules ${ }_{A\left(I^{\prime}\right)}$ with End $\left(\mathrm{Y}_{\mathrm{A}\left(\mathrm{I}^{\prime}\right)}\right)$ of finite representation type. Take any such tilting module $\mathrm{Y}$, considered as A-module, which does not contain an indecomposable injective A-module as direct summand, and form $P(I) \oplus \tau_{A}^{-1} Y_{A}$. Then clearly this is a tilting module, since it is the direct sum of $n$ pairwise non-isomorphic direct summands and satisfies the Ext-condition. Conversely, let $\mathrm{T}_{A}$ be a multiplicity-free tilting module with $\mathrm{B}=\operatorname{End}\left(\mathrm{T}_{\mathrm{A}}\right)$ of finite representation type, containing at least one indecomposable projective direct summand. A maximal projective direct summand of $T_{A}$ is of the form $P(I)$ for some non-empty subset $I \subseteq\{1, \ldots, n\}$. Let $\mathrm{T}_{\mathrm{A}}=\mathrm{P}(\mathrm{I})_{\mathrm{A}} \oplus \mathrm{X}_{\mathrm{A}}$. Now $\mathrm{X}$ has no non-zero projective direct summands, thus $X \approx \tau_{A}^{-1} \tau_{A} X$, and $\operatorname{Hom}(P(I), \tau X)=D \operatorname{Ext}(X, P(I))=0$, thus $\tau_{A} X$ is, in fact, an $A\left(I^{\prime}\right)$-module, and, of course, has no indecomposable injective A-module as direct summand. Also, End $\left(\tau_{A} X\right) \approx \operatorname{End}(X)$ is of the form eBe for some idempotent $e$, thus also End $\left(\tau_{A} X\right)$ is of finite representation type.

Let $\mathrm{T}_{\mathrm{A}}$ be a multiplicity-free tilting module with End $\left(\mathrm{T}_{A}\right)$ of finite representation type. Then we know that $\mathrm{T}_{\mathrm{A}}$ contains both non-zero preprojective and non-zero preinjective direct summands, thus only finitely many of the modules $\tau^{z_{T}}$, $z \in \mathbf{z}$ have $n$ indecomposable direct summands. Since any such class $\left\{\tau^{z_{T}}\right\}_{z}$ contains a tilting module with a non-zero projective direct summand, we conclude that alltogether there are only finitely many multiplicity-free tilting modules $T_{A}$ with End $\left(\mathrm{T}_{\mathrm{A}}\right)$ of finite representation type. 


\section{Tilting modules $\mathrm{T}_{\mathrm{A}}$ for $\mathrm{A}$ tame}

Lemma (3.1). Let $A$ be a hereditary tame algebra. Then there are only finitely many basic algebras of the form $B=$ End $\left(T_{A}\right)$, with $T_{A}$ a tilting module. Also, if $\mathrm{T}_{\mathrm{A}}$ is a tilting module, then $\mathrm{T}_{\mathrm{A}}$ has an indecomposable summand which is preprojectiv or preinjectiv.

Proof. Note that we may assume A to be connected. Let us first show the second assertion. A tilting module cannot be regular, since the dimension vectors of regular modules lie in a proper subspace of $G_{O}(A) \otimes Q$, namely in the hyperplane of vectors of zero defect.

However, the dimension vectors of the indecomposable direct summands of a tilting module generate $G_{0}(A) \otimes Q$. This shows that a tilting module has an indecomposable summand which is preprojective or preinjective.

Assume now that $\mathrm{T}_{\mathrm{A}}$ is a multiplicity free tilting module and contains a nonzero preprojective direct summand. Then there is $n \in \mathbb{N}$ such that $\tau^{n_{T}}$ has a nonzero projective direct summand, but $\tau^{i} T$ has no non-zero projective direct summand, for all $i<n$. Then $\tau^{n_{T}}$ also is a tilting module and $\operatorname{End}\left(T_{A}\right) \approx$ End $\left(\tau^{n} T\right)$. Thus we can assume that $T_{A}=e A \oplus T^{\prime}$ for some non-zero idempotent $e$ and a module $T^{\prime}$ without non-zero projective direct summands. Then $\operatorname{Hom}_{A}\left(e A, \tau^{-1} T^{\prime}\right)=0$, thus $\tau^{-1} T^{\prime}$ is an $\mathrm{A} /\langle e\rangle-$ module, and in fact an $\mathrm{A} /\langle\mathrm{e}\rangle-\mathrm{tilting}$ module. Since there are only finitely many $\mathrm{A} /\langle\mathrm{e}\rangle-\mathrm{tilting}$ modules, there are only finitely many multiplicity-free tilting modules $T_{A}$ with a non-zero projective direct summand.

Similarly, there are only finitely many multiplicity-free tilting modules $\mathbf{T}_{A}$ with a non-zero injective direct summand, and this gives all basic algebras which occur as endomorphism rings of tilting modules with a non-zero preinjective direct summand.

If $A$ is a hereditary algebra, we denote its quadratic form on $G_{0}(A)$ by $q$.

Proposition (3.2). Let $A$ be a connected tame hereditary algebra. Let $T_{A}$ be a tilting module and $B=$ End $\left(T_{A}\right)$. Then the following properties are equivalent:

(i) $T\left(T_{A}\right)$ is infinite.

(ii) $T\left(\mathrm{~T}_{\mathrm{A}}\right)$ contains infinitely many indecomposable preprojective modules, and the modules $\operatorname{Hom}_{A}\left({ }_{B} T_{A}, X_{A}\right)$ with $x_{A}$ indecomposable preprojective and in $T\left(T_{A}\right)$ form a component of the Auslander-Reiten quiver of $B$.

(iii) There exists a simple homogeneous module which belongs to $T\left(T_{A}\right)$.

(iv) A1l homogeneous modules belong to $T\left(T_{A}\right)$.

(v) If $R$ is indecomposable and regular, and $q($ dim $R)=0$, then there exists $i$ with $\tau{ }^{i} R$ in $T\left(T_{A}\right)$.

(vi) All preinjective modules belong to $T\left(T_{A}\right)$.

(vii) $T_{A}$ has no non-zero preinjective direct summand. 
Proof. The implications (ii) $\Rightarrow$ (i) and (iv) $\Rightarrow$ (iii) are trivial, the equivalence of (vi) and (vii) has been shown in lemma (2.2). Also, the implication (v) $\Rightarrow$ (iv) is obvious, since any homogeneous module $\mathrm{H}$ satisfies $\mathrm{q}$ (dim $\mathrm{H}$ ) $=0$ and $\tau \mathrm{H} \approx \mathrm{H}$.

(i) $\Rightarrow$ (vii): Assume $T_{A}$ has an indecomposable preinjective direct summand, say $\tau^{m} I$ for some $m \in \mathbb{N}$ and some indecomposable injective module $I$. Let $e$ be an idempotent of $A$ such that $e A / r a d(e A) \approx \operatorname{soc}(I)$. If $Y_{A}$ is generated by $T_{A}$, then $\operatorname{Ext}_{A}^{1}(T, Y)=0$, thus

$$
\begin{aligned}
0 & =\operatorname{Ext}_{A}^{1}\left(\tau^{m_{1}} I, Y\right) \approx D \operatorname{Hom}_{A}\left(Y, \tau^{m+1} I\right) \\
& \approx D \operatorname{Hom}_{A}\left(\tau^{-m^{-1}} Y, I\right) \\
& \approx \operatorname{Hom}_{A}\left(e A, \tau^{-m-1} Y\right)
\end{aligned}
$$

and therefore either $\mathrm{Y}$ is one of the finitely many indecomposable modules with $\tau^{-m^{-1}} \mathrm{Y}=0$, or else $\tau^{-\mathrm{m}^{-1}} \mathrm{Y}$ is indecomposable and one of the finitely many indecomposable $A /\left\langle e>-\right.$ modules, and $Y=\tau^{m+1} r^{-m-1} Y$. Altogether we see that there are only finitely many possibilities for $Y$.

(iii) $\Rightarrow$ (vii): Again, assume $\mathrm{T}_{\mathrm{A}}$ has an indecomposable preinjective direct summand $\tau^{\mathrm{m}} \mathrm{I}$. If $\mathrm{S}_{\mathrm{A}}$ is simple homogeneous, and in $T\left(\mathrm{~T}_{\mathrm{A}}\right)$, then

$$
0=\operatorname{Ext}_{A}^{1}\left(\tau^{m} I, S\right) \approx D \operatorname{Hom}\left(S, \tau^{m+1} I\right) \approx D \operatorname{Hom}(S, I) \text {, }
$$

since $\tau S \approx S$. However this contradicts the fact that dim $S$ has no zero component.

$$
\text { (vii) } \Rightarrow(v) \text { : Decompose } T_{A}=T^{\prime} \oplus T^{\prime \prime} \text {, with } T^{\prime} \text { preprojective and } T^{\prime \prime}
$$

regular. Let $R$ be indecomposable regular, with $q(\underline{\operatorname{dim}} R)=0$. Let $S$ be simple regular, with an epimorphism $R \rightarrow S$, and let $t$ be the $\tau$-period of $S$. Not all $\tau^{i} S, \quad 1 \leq i \leq t$, can be regular composition factors of $T^{\prime \prime}$, since $\operatorname{Ext}^{1}\left(T^{\prime \prime}, T^{\prime \prime}\right)=0$.

Thus, there is some $\tau^{j} S$ which is not a regular composition factor of $T "$. The top regular composition factor of $\tau^{j}{ }_{R}$ is $\tau^{j} S$, thus any non-trivial homomorphism $\tau^{j}{ }_{R} \rightarrow T^{\prime \prime}$ would imply that $T^{\prime \prime}$ has $\tau^{j} S$ as regular composition factor. Therefore

$$
\begin{aligned}
0 & =\operatorname{Hom}\left(\tau^{j} R, T^{\prime \prime}\right) \approx \operatorname{Hom}\left(\tau^{j+1} R, \tau T^{\prime \prime}\right) \\
& \approx D \operatorname{Ext}^{1}\left(T^{\prime \prime}, \tau^{j+1} R\right) .
\end{aligned}
$$

of course, we also have $\operatorname{Ext}^{1}\left(T^{\prime}, \tau^{j+1} R\right)=0$, since $T^{\prime}$ is preprojective and $\tau^{j+1} R$ is regular. Thus $\operatorname{Ext}^{1}\left(T, \tau^{j+1} R\right)=0$, and therefore $\tau^{j+1} R \in T\left(T_{A}\right)$.

(iv) $\Rightarrow$ (ii): Let $\mathrm{S}$ be a simple homogeneous module. Now, no indecomposable regular module with regular composition factors $\mathrm{S}$ can be in add $\mathrm{T}_{\mathrm{A}}$, since $\operatorname{Ext}^{1}(S, S) \neq 0$. Note that $\operatorname{Hom}(X, S) \neq 0$ for a module $X$, implies that $X$ has an indecomposable direct summand which is either preprojective or regular with regular composition factors $S$. Since $S$ is generated by $T_{A}$, we conclude that ${ }_{A}$ has an indecomposable preprojective direct summand; in particular, there exists an indecomposable preprojective module $x$ in $T\left(T_{A}\right)$. Now, given $X$ indecomposable preprojective and in $T\left(T_{A}\right)$, we want to construct an indecomposable preprojective module $X^{\prime}$ 
in $T\left(T_{A}\right)$ with a non-zero and non-invertible map $X \rightarrow X^{\prime}$. Note that $\operatorname{Ext}^{1}(S, X)=D \operatorname{Hom}(X, \tau S) \neq 0$, since $S \approx \tau S$ satisfies $\operatorname{Hom}(P, S) \neq 0$ for all indecomposable projective modules $P$, and $X \approx \tau^{-n} P$ for some $n \in \mathbb{N}$, and some $P$. Let

$$
\mathrm{O} \longrightarrow \mathrm{X} \stackrel{\mathrm{m}}{\rightarrow} \mathrm{E} \stackrel{\mathrm{P}}{\rightarrow} \mathrm{S} \rightarrow 0
$$

be a non-split exact sequence. No direct summand of $E$ can be isomorphic to $S$, since otherwise the sequence would split. Thus $E$ has to be preprojective. Also, with $X$ and $S$ also $E \in T\left(T_{A}\right)$. Thus, for $X^{\prime}$ we may take any indecomposable direct summand of $\mathrm{E}$.

Next, assume $X$ is indecomposable preprojective and in $T\left(T_{A}\right), Y$ an indecomposable module in $T\left(T_{A}\right)$, and there is a given a map $f: X \rightarrow Y$ with $\operatorname{Hom}_{A}\left({ }_{B} T_{A}, f\right)$ irreducible in $M_{B}$. We claim that $Y$ also has to be preprojective. First, assume $Y$ is regular. Choose a simple homogeneous module $S$ which is not a regular composition factor of $Y$, and form a non-split exact sequence (*).

The exact sequence induced by $f$ must split, since $\operatorname{Ext}^{1}(S, Y)=0$, thus $f$ can be factored as $f=f^{\prime} m$ through $E$ and neither $m$ is split mono, nor $f^{\prime}$ is split epi. Since $E \in T\left(T_{A}\right)$, we obtain a factorization of $\operatorname{Hom}_{A}\left({ }_{B} T_{A}, f\right)$ with the same properties, thus $\operatorname{Hom}_{A}\left({ }_{B} T_{A}, f\right)$ cannot be irreducible. Similarly, if $Y$ is preinjective, then any simple homogeneous module $S$ satisfies $E^{1}(S, Y)=0$, thus again $\operatorname{Hom}_{A}\left({ }_{B} T_{A}, f\right)$ cannot be irreducible. It follows now that the set $C$ of $B$-modules of the form $\operatorname{Hom}_{A}\left({ }_{B} T_{A}, X_{A}\right)$ with $x_{A}$ indecomposable projective and in $T\left(T_{A}\right)$ is a complete component of the Auslander-Reiten quiver of $B$. Namely, assume there is an irreducible map $\mathrm{N}_{B} \rightarrow \mathrm{N}_{B}^{\prime}$ with $\mathrm{N}_{B}$ in $C$, and $\mathrm{N}_{B}^{\prime}$ indecomposable. Now, either $N_{B}^{\prime}=\operatorname{Hom}_{A}\left(T_{A}, Y_{A}\right)$ or $=\operatorname{Ext}_{A}^{1}\left({ }_{B} T_{A}, Y_{A}\right)$ for some indecomposable module $Y_{A}$. In the second case, the irreducible map $\mathrm{N}_{B} \rightarrow \mathrm{N}_{B}^{\prime}$ is involved in a connecting sequence and $\mathrm{N}_{B} \approx \operatorname{Hom}_{A}\left({ }_{B} T_{A}, I_{A}\right)$ for some indecomposable injective module, contrary to the assumption $N_{B} \in C$. Thus $N_{B}^{\prime}=\operatorname{Hom}_{A}\left({ }_{B} T_{A}, Y_{A}\right)$, and then, by the previous considerations, $Y_{A}$ is preprojective.

Proposition $(3.2)^{*}$. Let $A$ be a connected tame hereditary algebra, and $T_{A}$ a tilting module with $B=$ End $\left(T_{A}\right)$. Then the following properties are equivalent:

(i) $F\left(T_{A}\right)$ is infinite.

(ii) $F\left(\mathrm{~T}_{\mathrm{A}}\right)$ contains infinitely many indecomposable preinjective modules, and the modules $\operatorname{Ext}_{A}^{1}\left({ }_{B} T_{A}, X_{A}\right)$ with $x_{A}$ indecomposable preinjective and in $F\left(T_{A}\right)$ form a component of the Auslander-Reiten quiver of $B$.

(iii) There exists a simple homogeneous module which belongs to $F\left(T_{A}\right)$.

(iv) Al1 homogeneous modules belong to $F\left(T_{A}\right)$.

(v) If $R$ is indecomposable and regular, and $q$ (dim $R$ ) $=0$, then there exists $i$ with $\tau^{i}$ in $F\left(T_{A}\right)$.

(vi) All preprojective modules belong to $F\left(T_{A}\right)$. 
(vii) $T_{A}$ has no non-zero preinjective direct summand.

Proof. The proof is similar to the previous one, again the implications (ii) $\Rightarrow$ (i), (iv) $\Rightarrow$ (iii) and (v) $\Rightarrow$ (iv) are obvious, and the equivalence of (vi) and (v) follows from (2.2).

(i) $\Rightarrow$ (vii), and (iii) $\Rightarrow$ (vii): Let $\mathrm{P}$ be indecomposable preprojective,

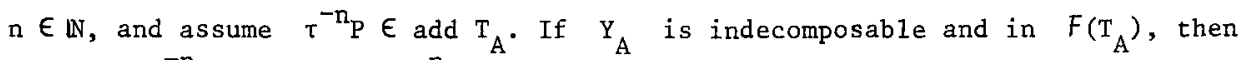
$0=\operatorname{Hom}_{A}\left(\tau^{-n_{1}} P, Y\right) \approx \operatorname{Hom}_{A}\left(P, \tau^{n_{Y}}\right)$, thus there are only a finite number of possibilities

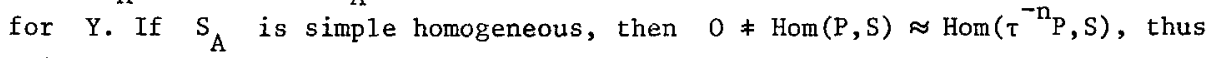
$S \notin F\left(\mathrm{~T}_{\mathrm{A}}\right)$.

(vii) $\Rightarrow(v)$ : Decompose $T_{A}=T^{\prime \prime} \oplus T^{\prime \prime \prime}$ with $T^{\prime \prime}$ regular and $T^{\prime \prime \prime}$ preinjective. Let $R$ be indecomposable regular, with $q($ dim $R)=0$, let $S$ be a simple regular submodule of $R$, say with $\tau$-period $t$. Since Ext ${ }^{1}\left(T^{\prime \prime}, T^{\prime \prime}\right) \neq 0$, there exists some $i$, such that $\tau^{i} S$ is not a regular composition factor of $T$ ". Then any homomorphism $\varphi: T^{\prime \prime} \rightarrow \tau \tau^{i_{R}}$ is zero, since otherwise the image would contain $\tau^{j}$, and $\tau^{j} S$ would appear as regular composition factor of $T^{\prime \prime}$. Thus $\operatorname{Hom}\left(T^{\prime \prime}, \tau{ }^{i} R\right)=0$, and of course, also $\operatorname{Hom}\left(T^{\prime \prime}, \tau^{\left.i_{R}\right)}=0\right.$, thus $\tau_{R} \in F\left(T_{A}\right)$.

(iv) $\Rightarrow$ (ii): Let $S$ be simple homogeneous. Since $S \in F\left(T_{A}\right)$, we know that $S$ is cogenerated by $\tau \mathrm{T}_{\mathrm{A}}$, and this immediately implies that $\tau \mathrm{T}_{\mathrm{A}}$ has an indecomposable preinjective direct summand, which clearly has to belong to $F\left(\mathrm{~T}_{A}\right)$. Now, given any indecomposable preinjective module $X \in F\left(T_{A}\right)$. Then $\operatorname{Ext}^{1}(X, S) \neq 0$, since $S$ is homogeneous, and $x$ preinjective, thus consider a non-split extension.

$$
\mathrm{O} \rightarrow \mathrm{S} \stackrel{\mathrm{m}}{\rightarrow} \mathrm{E} \stackrel{\mathrm{p}}{\longrightarrow} \mathrm{X} \longrightarrow 0 \text {. }
$$

Clearly $E$ has to be preinjective and to belong to $F\left(T_{A}\right)$. In this way, we construct inductively infinitely many indecomposable preinjective modules in $F\left(T_{A}\right)$.

Let $\mathrm{X}$ be indecomposable preinjective and in $F\left(T_{A}\right)$, and $Y$ is indecomposable in $F\left(T_{A}\right)$, and assume then is given a map $f: Y \rightarrow X$ such that $\operatorname{Ext}_{A}^{1}\left({ }_{B} T_{A}, f\right)$ is irreducible in $M_{B}$. Then $Y$ has to be preinjective. Namely, assume $Y$ is regular or preprojective, let $S$ be simple homogeneous which, in case $Y$ is regular, is not a regular composition factor of $Y$. Then $\operatorname{Ext}^{1}(Y, S)=0$, thus the map $f$ can be lifted to $E$ : there exists $f^{\prime}: Y \rightarrow E$ with $f=p f^{\prime}$. This is a non-trivial factorization of $f$, and therefore we obtain also a non-trivial factorization of $\operatorname{Ext}_{A}^{1}\left({ }_{B}{ }^{T} A, f\right)$, contrary to the assumption that $\operatorname{Ext}_{A}^{1}\left({ }_{B} T_{A}, f\right)$ is irreducible. This shows that the set of modules $\operatorname{Ext}_{A}^{1}\left({ }_{B} T_{A}, X_{A}\right)$ with $X_{A}$ indecomposable preinjective and in $F\left(T_{A}\right)$ is closed under irreducible maps, taking into account that the only indecomposable $B$-modules which are of the form $\operatorname{Ext}{ }_{A}^{1}\left({ }_{B} T_{A}, X_{A}\right)$ with $X_{A}$ indecomposable and also are codomains of irreducible maps with domain $\operatorname{Hom}_{A}\left({ }_{B} T_{A}, Y_{A}\right)$ are the modules $\operatorname{Ext}_{A}^{1}\left({ }_{B} T_{A}, P_{A}\right)$ with $P$ indecomposable projective, and then $X_{A} \approx P_{A}$, thus $X_{A}$ is not preinjective. 
(3.3) We now can distinguish the following possibilities for a tilting module $T_{A}$. Always, A denotes a connected tame hereditary algebra, and $B=E n d\left(T_{A}\right)$. (I) $\quad \mathrm{T}_{\mathrm{A}}$ is preprojective or $\mathrm{T}_{\mathrm{A}}$ is preinjective.

(II) $\mathrm{T}_{\mathrm{A}}=\mathrm{T}^{\prime} \oplus \mathrm{T}^{\prime \prime}$ with $\mathrm{T}^{\prime} \neq 0$ preprojective and $\mathrm{T}^{\prime \prime} \neq 0$ regular.

(II*) $\quad \mathrm{T}_{\mathrm{A}}=\mathrm{T}^{\prime \prime} \oplus \mathrm{T}^{\prime \prime}$ with $\mathrm{T}^{\prime \prime} \neq 0$ regular and $\mathrm{T}^{\prime \prime \prime} \neq 0$ preinjective.

(III) $\quad \mathrm{T}_{\mathrm{A}}=\mathrm{T}^{\prime} \oplus \mathrm{T}^{\prime \prime} \oplus \mathrm{T}^{\prime \prime \prime}$ with $\mathrm{T}^{\prime} \neq 0$ preprojective, $\mathrm{T}^{\prime \prime}$ regular, and $\mathrm{T}^{\prime \prime \prime} \neq 0$ preinjective.

It is clear that these cases are mutually disjoint. Also, they exhaust all possibilities, since according to 3.1 , a tilting module cannot be regular. Let us consider the various cases in more detail.

Case (I). The endomorphism algebras of preprojective modules can also be obtained as endomorphism algebras of preinjective modules, and vice versa. Namely, for any $m$, the full subcategory of all preprojective modules $X$ satisfying $\tau^{m} X=0$ is equivalent to the full subcategory of all preinjective modules $Y$ satisfying $\tau^{-m_{Y}}=0$ (this follows from the explicit description of these categories given in [2]). Thus, being interested in tilted algebras, we may restrict, in case (I), to the endomorphism algebras of preprojective tilting modules.

Let $\mathrm{T}_{\mathrm{A}}$ be a preprojective tilting module. If an indecomposable A-module $\mathrm{X}$ is not in $T\left(T_{A}\right)$, then it is a predecessor of one of the indecomposable direct sum mands of $\tau T_{A}$. [Namely, $X$ has a non-zero factor module in $F\left(T_{A}\right)$, thus $\left.\operatorname{Hom}_{A}\left(X, \tau T_{A}\right) \neq 0.\right]$ In particular, the set $Z$ of indecomposable $A$-modules not in $T\left(T_{A}\right)$ is finite and contains only preprojective modules. Of course, the indecomposable A-modules in $F\left(T_{A}\right)$ belong to $Z$.

The Auslander-Reiten quiver of $B$ is obtained from that of $A$ by deleting the finitely many modules from $Z$, and using the relative Auslander-Reiten sequences from $T\left(T_{A}\right)$ and joining to the component of preinjective A-modules the indecomposable modules from $F\left(T_{A}\right)$, starting with the connecting sequences and continuing with the relative Auslander-Reiten sequences inside $F\left(\mathrm{~T}_{\mathrm{A}}\right)$.




$\Gamma(B)$

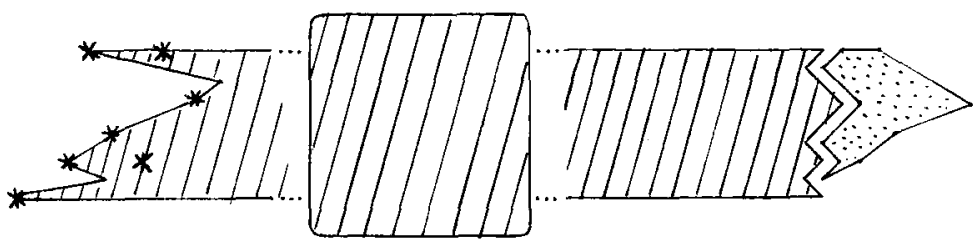

It is clear that the endomorphism algebras $B=$ End $\left(T_{A}\right)$ of preprojective tilting modules are "concealed hereditary algebras" in the sense of [6], the equivalent cofinite full subcategories of $M_{A}$ and $M_{B}$ being given by $T\left(T_{A}\right)$ and its image under $\operatorname{Hom}_{A}\left({ }_{B} T_{A},-\right)$.

Case (II). This is situation 3.2, thus $T\left(T_{A}\right)$ contains infinitely many indecomposable preprojective modules, and their images under $F=\operatorname{Hom}_{A}\left({ }_{B} T_{A},-\right)$ form a complete connected component of $\Gamma(B)$. Also $T\left(T_{A}\right)$ contains all preinjective modules, and as usual, their images under $F$ form only part of a component $C$ of $\Gamma(B)$, we have to add the indecomposable modules of the form $\operatorname{Ext}_{A}^{1}\left({ }_{B} T_{A}, P\right)$, with $P$ projective, and close under relative Auslander-Reiten sequences from $F\left(T_{A}\right)$. According to $3.2^{*}$, the category $F\left(T_{A}\right)$ is finite, thus $C$ will consist precisely of the images of the indecomposable preinjective modules under $F$ and the images of the indecomposable modules in $F\left(T_{A}\right)$ under $\operatorname{Ext}_{A}^{1}\left({ }_{B} T_{A}\right.$, - ). The remaining components of $\Gamma(B)$ correspond bijectively to the regular components of $\Gamma(A)$. In fact, the homogeneous components of $\Gamma(A)$ are preserved under $F$, since all homogeneous modules belong to $T\left(\mathrm{~T}_{\mathrm{A}}\right)$. The remaining regular components of $\Gamma(A)$ may contain also indecomposable modules not in $T\left(T_{A}\right)$, these have to be deleted - however, according to $3.2(v), T\left(T_{A}\right)$ contains quite a few modules from any regular component (of course, this also follows from the fact that all components of $\Gamma(B)$ have to be infinite).

$\Gamma(\mathrm{A})$

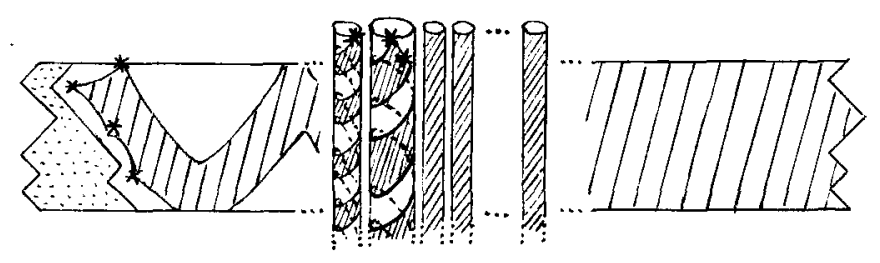

$\Gamma(B)$




Clearly, the endomorphism algebras $B=$ End $\left(\mathrm{T}_{\mathrm{A}}\right)$, with $\mathrm{T}_{\mathrm{A}}$ of type (II), are "domestic regular enlargements" (in the sense of [6]) of a tame concealed hereditary algebra $C$. Here, $C$ is given by the algebra $C=$ End $\left(T_{A}^{\prime}\right)$.

Case (II*): This case is dual to the previous case (II), we obtain as endomorphism algebra a "domestic regular coenlargement" of a tame concealed hereditary algebra.

Case (III): According to 3.2 and $3.2^{*}$, both $T\left(\mathrm{~T}_{\mathrm{A}}\right)$ and $F\left(\mathrm{~T}_{\mathrm{A}}\right)$ are finite categories, thus $B=\operatorname{End}\left(T_{A}\right)$ is of finite representation type. 


\section{Examples}

\section{(4.1) A of type $A_{n}$}

First, let us consider only tilting modules $\mathrm{T}_{\mathrm{A}}$ such that the endomorphism ring End $\left(T_{A}\right)$ has an indecomposable faithful module.

Lemma. Let $A$ be of type $A_{n}$, and $T_{A}$ a tilting module, and assume that $B=\operatorname{End}\left(T_{A}\right)$ has an indecomposable faithful module. Then $T_{A}$ is a slice module (thus, $B$ is hereditary of type $A_{n}$, again).

Proof. We may consider the Auslander-Reiten quiver $\Gamma(A)$ of $A$ as a subset of $\mathbb{Z A}_{\mathrm{n}}$, the elements of $\mathbb{Z A}_{\mathrm{n}}$ being indexed as follows:



We will use, as in [3], rectangles of the form $q_{(z, r)}=\left\{\left(z^{\prime}, r^{\prime}\right) \mid z+r-n \leq z^{\prime} \leq z\right.$, $\left.z+1 \leq z^{\prime}+r^{\prime} \leq z+r\right\}$ for some fixed $(z, r) \in \mathbb{Z} A$, and will call $q(z, r)$ the rectangle ending at $(z, r)$, or starting at $(z+r-n, n-r+1)$, and also write $(z+r-n, n-r+1)^{q}$ instead of $q_{(z, r)}$.



The importance of these rectangles lies in the following facts: let $X, Y$ be indecomposable representations of $A$. Then

$$
\operatorname{Hom}(X, Y) \neq 0 \quad \text { iff } \quad \underline{\operatorname{dim}} X \in q_{\text {dim } Y} \text {, and, of course, this is }
$$

equivalent to $\underline{\operatorname{dim}} Y \in \underline{\operatorname{dim} X} \mathrm{q}$.

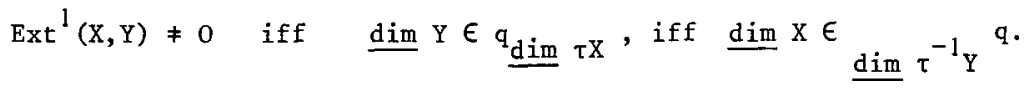

Now assume $T_{A}$ is a tilting module such that $\operatorname{End}\left(\mathrm{T}_{A}\right)$ has an indecomposable faithful module. Applying, if necessary, some $\tau^{*}$, we may assume that $T_{A}$ has a non-zero projective direct sumnand, and this implies that any indecomposable faithful $B$-module, with $B=\operatorname{End}\left(T_{A}\right)$, is of the form $\operatorname{Hom}_{A}\left({ }_{B}{ }^{T}{ }_{A}, X_{A}\right)$ for some indecomposable module $X_{A}$. Note that the fact that $\operatorname{Hom}_{A}\left({ }_{B} T_{A}, X_{A}\right)$ is faithful means that 
$\operatorname{Hom}_{A}\left(T(i)_{A}, X_{A}\right) \neq 0$ for any indecomposable direct summand $T(i)$ of $T_{A}$. Let $x=\underline{\operatorname{dim}} x, t(i)=\underline{\operatorname{dim}} T(i)$, thus $t(i) \in q_{x}$ for all $x$. In case there exists a sequence of non-zero maps

$$
\mathrm{T}(\mathrm{i})=\mathrm{T}\left(\mathrm{i}_{1}\right) \rightarrow \mathrm{T}\left(\mathrm{i}_{2}\right) \rightarrow \cdots \rightarrow \mathrm{T}\left(\mathrm{i}_{\mathrm{s}}\right)=\mathrm{T}(\mathrm{j}),
$$

then clearly $t(j)$ belongs to the set $\left\{\left(z^{\prime}, r^{\prime}\right) \mid z \leq z^{\prime}, z+r \leq z^{\prime}+r^{\prime}\right\}$ where $t(i)=(z, r)$, thus $t(j) \in q_{x} \cap t(i)^{q}$.

Now, let $T\left(i_{1}\right), \ldots, T\left(i_{u}\right)$ be the sources of add $T_{A}$. Then, by the previous considerations, al1 $t(j)$ belong to $q_{x} \cap \bigcup_{\alpha=1}^{u} t\left(i_{\alpha}\right)^{q}$, and since $\operatorname{Ext}\left(T(j), T\left(i_{\alpha}\right)\right)=0$ for a11 $\alpha$, we also have $t(j) \notin \bigcup_{\alpha=1}^{u} \tau_{t\left(i_{\alpha}\right)}^{-1} q$.



We see that from any $\tau$-orbit of $Z \mathbb{A}_{n}$, at most one element can be of the form $t(j)$, thus, since there are precisely $n$ different $t(j)$, it follows that $\{T(j) \mid j\}$ is a complete slice.

Let us classify now all tilting modules $T_{A}$, for $A=A(n)$ the path algebra

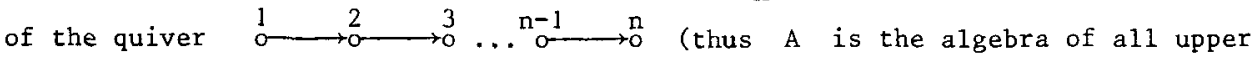
triangular $n \times n-m a t r i c e s$, for some $n$ ).

Lemma. Let $d=\left(d_{1}, \ldots, d_{n}\right)$ be an integral vector with $d_{i}>0$ for all i. Then there exists a tilting module of dimension type $d$ if and only if for any pair $i<k$ with $d_{i}=d_{k}$, there exists $j$ with $i<j<k$ and $d_{i}>d_{j}$.

Proof. Suppose d does not satisfy this condition. Then it is easy to construct a non-trivial semi-invariant on the affine space of all A-modules of dimension type $d$, thus there cannot be a tilting module of dimension type $d$, see [4].

Conversely, assume the condition is satisfied, and 1 et $d_{j}=\underset{1 \leq i \leq n}{\min } d_{i}$. Let $\mathrm{T}(1)$ be the unique indecomposable representation of dimension type $\ell=(1, \ldots, 1)$, note that $T(1)$ is both projective and injective, and let $d^{\prime}=d-d_{j} l l$. The support of $d^{\prime}$ has precisely $n^{-l}$ elements, and the restriction of d' to its support satisfies the corresponding condition, thus, by induction, there is a module $T_{A}^{\prime}$ with $\operatorname{dim} T_{A}^{\prime}=d^{\prime}$ and $\operatorname{Ext}_{A}^{1}\left(T_{A}^{\prime}, T_{A}^{\prime}\right)=0$. Also, by induction, the number of isomorphism classes of indecomposable direct summands of $T^{\prime}$ is precisely $n^{-1}$. It follows that $T_{A}=\left(d_{j}^{\oplus} T(1)\right) \oplus T_{A}^{\prime}$ is a tilting module. 
The proof above gives a complete description of all tilting modules. Of course, we may restrict to multiplicity-free ones: Recall that we may associate to any indecomposable module $X_{A}$, an interval $\left[\alpha_{X}, \beta_{X}\right]$, namely the support of dim $X$ in $\{1,2, \ldots, n\}$. Let $\alpha_{i}=\alpha_{T(i)}, \beta_{i}=\beta_{T(i)}$. The interval $\left[\alpha_{i}, \beta_{i}\right]$ are obtained inductively by deleting in any given interval one point, and considering the remaining points: they form either one or two new intervals: one interval, in case the deleted point was an end point, and two interval otherwise. There is an epimorphism $\mathrm{T}(\mathrm{i}) \rightarrow \mathrm{T}(\mathrm{j})$ if and only if $\alpha_{i}=\alpha_{j}$ and $\beta_{i} \geq \beta_{j}$, and there is an monomorphism $T(i) \rightarrow T(j)$ if and only if $\alpha_{i} \geq \alpha_{j}$ and $\beta_{i}=\beta_{j}$. Altogether, we see that the endomorphism ring $B=\operatorname{End}\left(\mathrm{T}_{A}\right)$ of $\mathrm{T}_{A}$ is a full connected subquiver of the following "genealogical" tree



with all possible relations $\varphi \psi=0$. Conversely, given the path algebra of a full connected subquiver of the genealogical tree containing $n$ points, with all relations $\varphi \psi=0$, there is a unique multiplicity-free tilting module with this algebra as endomorphism algebra.

\section{(4.2) $A$ of type $E_{6}$.}

The following list contains all tilted algebras $B=$ End $\left(T_{A}\right)$, where $A$ is hereditary of type $E_{6}$, and $T_{A}$ a tilting module, such that there exists an indecomposable faithful B-module. We have written down the quiver with relation of $B$. As relations, one always has all possible commutativity relations, and some additional zero-relations indicated by a dotted line joining the starting point and the end point of the relation. Non-oriented arrows can be oriented arbitrarily.
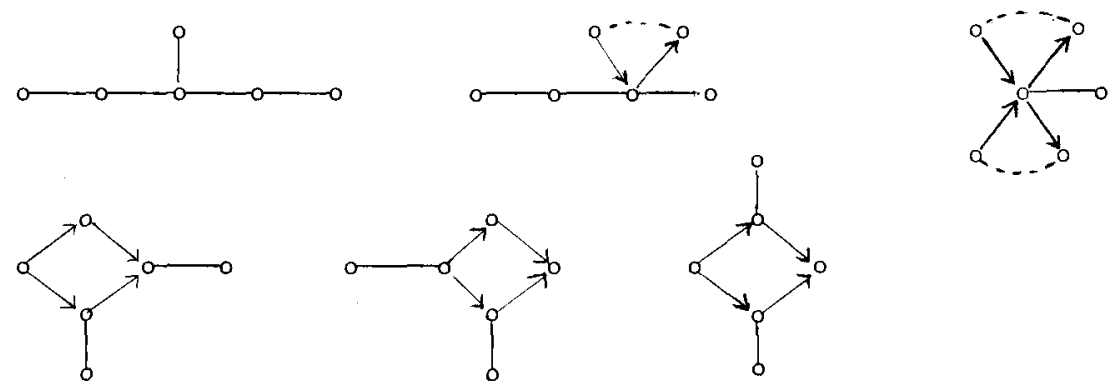


<smiles>O=C1OOOO1</smiles><smiles>o1oooooo1</smiles>
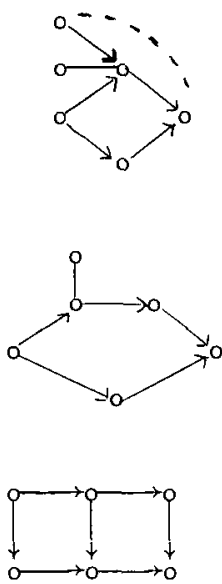
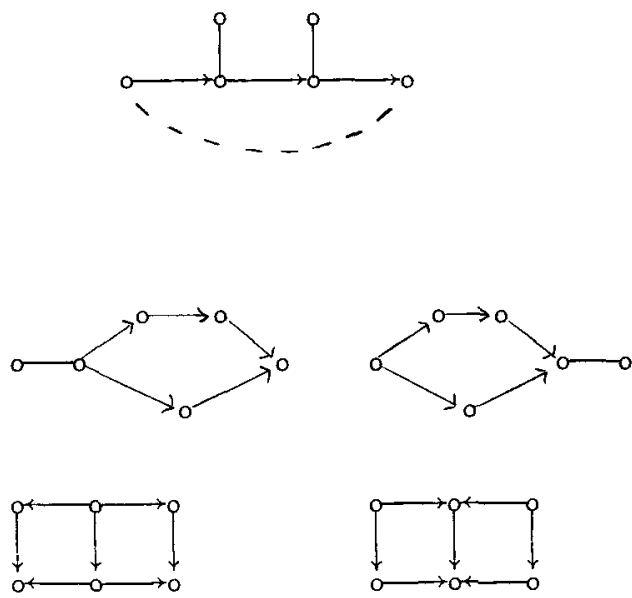

\section{(4.3) A of type $\widetilde{A}_{n}$.}

Lemma. Let $A$ be of type $\widetilde{A}_{n}$, and $T_{A}$ a preprojective tilting module. Then $\mathrm{T}_{\mathrm{A}}$ is a slice module.

Proof. Without loss of generality, we may assume that all simple projective A-modules are direct summands of ${ }^{T} A$, applying, if necessary, a suitable number of reflection functors. Let $a_{1}, \ldots, a_{r}$ be the sinks, and $b_{1}, \ldots, b_{r}$ the sources of the quiver of A. Since $P\left(a_{j}\right)$ is a direct summand of $T_{A}$, for $1 \leq j \leq r$, we have

$$
0=\operatorname{Ext}^{\prime}\left(T(i), P\left(a_{j}\right)\right)=D \operatorname{Hom}\left(P\left(a_{j}\right), \tau T(i)\right)
$$

for all direct summands $T(i)$ of $T_{A}$. The defect $\partial(\tau T(i))$ of $\tau T(i)$ can be calculated as follows:

$$
\partial(\tau T(i))=\sum_{j=1}^{r} \operatorname{dim} \operatorname{Hom}\left(P\left(b_{j}\right), \tau T(i)\right)-\sum_{j=1}^{r} \operatorname{dim} \operatorname{Hom}\left(P\left(a_{j}\right), \tau T(i)\right) \geq 0,
$$

thus $\tau T(i)$ can only be preprojective in case $\tau T(i)=0$. As a consequence, all $\mathrm{T}(\mathrm{i})$ are projective, and therefore $\mathrm{T}_{\mathrm{A}}$ is the slice module of a complete slice.

\section{(4.4) A of type $\widetilde{\mathrm{E}}_{6}$.}

The following list contains all tilted algebras $B=\operatorname{End}\left(T_{A}\right)$, where $A$ is of type $\widetilde{\mathrm{E}}_{6}$ and $\mathrm{T}_{A}$ is a preprojective tilted algebra (again, given by the quiver of B, with all commutativity relations and additional zero relations).
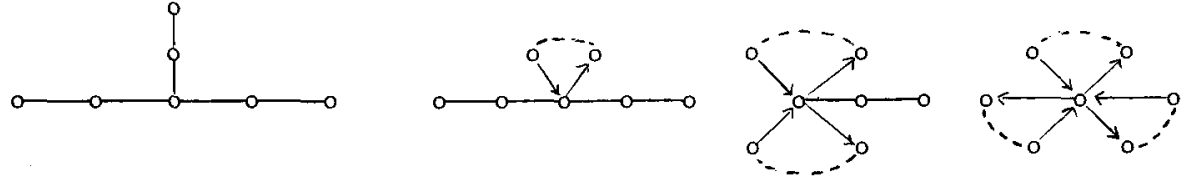

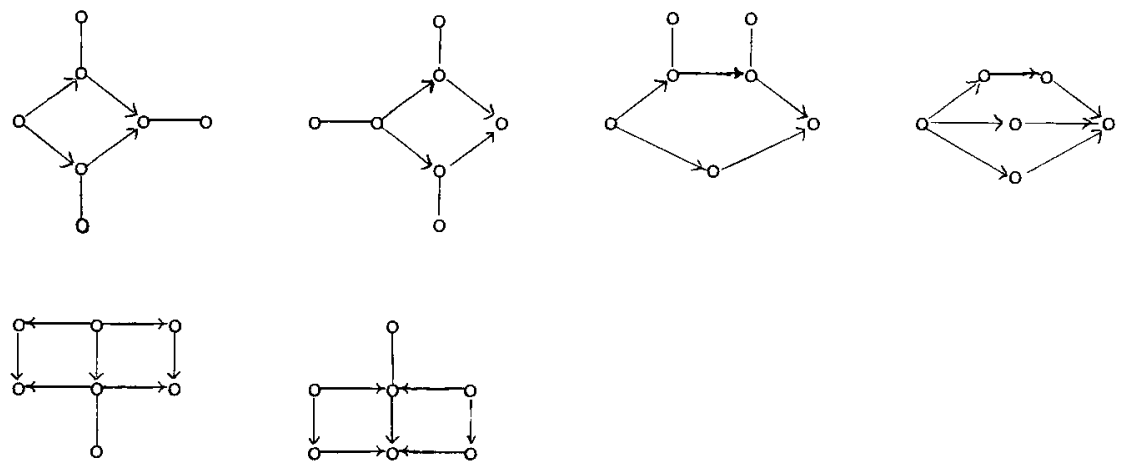
[1] D1ab, V., Ringel, C.M.: Indecomposable representations of graphs and algebras. Memoirs Amer. Math. Soc. 173 (1976).

[2] Dlab, V., Ringel, C.M.: The preprojective algebra of a modulated graph. Proceedings Ottawa Conference 1979. To appear (Springer Lecture Notes).

[3] Gabriel, P., Riedtmann, Chr.: Group representations without groups. Comment. Math. Helv. 54 (1979), 240-287.

[4] Happel, D.: Relative invariants and subgeneric orbits of quivers of finite and tame type.I. To appear.

[5] Happe1, D., Ringel, C.M.: Tilted algebras. To appear.

[6] Ringe1, C.M. Tame algebras. Proceedings Ottawa Conference 1979. To appear (Springer Lecture Notes).

D. Happel

C.M. Ringel

Fakultät für Mathematik

Universität Bielefeld

Universitätsstr. 1

D - 4800 Bielefeld I

West-Germany . 